\title{
Parametrização de campos de velocidades sísmicas por série ondaleta Haar usando algoritmo piramidal e técnicas de redução de parâmetros
}

\author{
Alexsandro G. Cerqueira*, UFBA, Salvador, BA, Brasil
}

Wilson M. Figueiró, CPGG-IGEO-UFBA, Salvador, BA, Brasil

Paulo E. M. Cunha, CENPES, Petrobras, Rio de Janeiro, RJ, Brasil

Copyright 2014, SBGf - Sociedade Brasileira de Geofísica

Este texto foi preparado para a apresentação no VI Simpósio Brasileiro de Geofísica, Porto Alegre, 14 a 16 de outubro de 2014. Seu conteúdo foi revisado pelo Comitê Técnico do VI SimBGf, mas não necessariamente representa a opinião da SBGf ou de seus associados. É proibida a reprodução total ou parcial deste material para propósitos comerciais sem prévia autorização da SBGf.

\begin{abstract}
The representation of compressional seismic velocity fields, originated by geological model through numerical parameters, is extremely important in geophysics, because it makes possible the quantification of the mentioned property of such qualitative geological models, so they can be mathematically manipulated. The parameterization by Haar wavelet series is seen like an attractive alternative to quantify such aspects of geological models. The Haar Wavelet Discrete Transform can be used to obtain those parameters and it helps in the application of a filter that assures a reasonable parameterization with few coefficients. The wavelet series expresses satisfactorily complicated functions through linear combination of simpler functions. The pyramidal algorithm appears as a synthetic way to calculate coefficients. Some techniques are applied in order to reduce the number of coefficients used in the parameterization.
\end{abstract}

Key words: wavelet series, pyramidal algorithm, coefficient reduction, filtering and parameterization.

\section{Resumo}

A representação de campos de velocidades sísmicas compressionais, oriundos de modelos geológicos, através de parâmetros numéricos é de extrema importância na geofísica, pois torna possível a quantificação da mencionada propriedade de tais modelos, estes vistos inicialmente em termos puramente qualitativos, permitindo assim que sejam matematicamente manipulados. A parametrização por série ondaleta Haar é vista como uma alternativa atrativa para quantificar tais modelos geológicos. A Transformada Discreta Ondaleta Haar pode ser utilizada para a obtenção de tais parâmetros e também auxiliar na aplicação de um filtro que garanta uma parametrização razoável com poucos coeficientes. A série ondaleta representa, satisfatoriamente, funções complicadas através de combinações lineares de funções mais simples. O algoritmo piramidal aparece como um modo sintético de cálculo de coeficientes. Algumas técnicas de filtragem são aplicadas com o intuito de diminuir o número de coeficientes utilizados na parametrização.

Palavras chaves: série ondaleta, algoritmo piramidal, redução de coeficientes, filtragem e parametrização.

\section{Introdução}

Na geofísica, existe a preocupação de tornar quantitativos modelos qualitativos provenientes da geologia de modo a torná-los matematicamente manipuláveis. Para tanto, utiliza-se a parametrização, que dentre alguns tipos mais conhecidos, pode-se citar: a matricial, a polinomial (dos Santos \& Figueiró, 2011), a trigonométrica, a por splines e outras.

Uma das formas, já conhecida, de parametrizar propriedades de modelos geológicos é a série ondaleta. Como exemplo, tem-se a série ondaleta Haar (Martinez \& Figueiró, 2011; Perin \& Figueiró, 2012; e Cerqueira \& Figueiró, 2013) que representa funções quadrado integráveis através de combinação linear de funções pertencentes a uma base mais simples, onde os coeficientes da combinação são os parâmetros a serem estimados em procedimentos de inversão.

Uma forma simples de se obter os coeficientes utilizados na série ondaleta de Haar é através o algoritmo piramidal (Cunha, 2010), também conhecido como Transformada Discreta Ondaleta, que, por meio de manipulações numéricas, permite representar campos de velocidades sísmicas pela referida série.

Neste trabalho, é realizada a parametrização de campos de velocidades sísmicas compressionais de modelos geológicos simples, previamente conhecidos, utilizando a série ondaleta Haar, cujos coeficientes são obtidos através do algoritmo piramidal que auxilia técnicas de redução de coeficientes que são aplicadas com o objetivo de diminuir ao máximo o número de coeficientes utilizados em cada parametrização.

\section{Aspectos Teóricos}

A partir de uma função ondaleta, $\psi$, uma base é definida como aquela constituida por funções do tipo $\psi_{j, k}$, chamadas de ondaletas filhas. A representação por série ondaleta considera dilatações (compressões) e translações dessa única função, $\psi$, de modo a obter uma aproximação da função que se deseja representar. Tendo em vista a ortonormalidade, as funções da mencionada base devem obedecer à seguinte forma (Morettin, 1999):

$\psi_{j, k}(t)=2^{j / 2} \psi\left(2^{j} t-k\right)$, onde $j$ e $k \in Z$.

O sistema ortonormal $\left(\psi_{j, k}\right)$ forma uma base de $L^{2}(R)$, espaço das funções reais de variável real quadrado integráveis. Existem coeficientes $c_{j, k}$ que tornam possível representar uma função $f(t)$ através da série ondaleta:

$f(t)=\sum_{j=-\infty}^{\infty} \sum_{k=-\infty}^{\infty} c_{j, k} \cdot \psi_{j, k}(t)$.

Os coeficientes $\left(c_{j, k}\right)$ são dados por: 
$c_{j, k}=\left\langle f \mid \psi_{j, k}\right\rangle=\int_{-\infty}^{\infty} f(t) \cdot \psi_{j, k}(t) d t$

Nem todas as ondaletas geram sistemas ortogonais. Nesse caso, é necessário gerar ondaletas através da função escala, $\phi$, também chamada de função indicatriz:

$\phi(\mathrm{t})=\sqrt{2} \sum_{k} l_{k} \phi(2 \mathrm{t}-\mathrm{k})$,

que permite gerar a familia ortogonal em $L^{2}(R)$ :

$\phi_{\mathrm{j}, \mathrm{k}}(\mathrm{t})=2^{\mathrm{j} / 2} \phi\left(2^{j} t-k\right)$, onde $j$ e $k \in Z$.

O sistema ortonormal:

$\left\{\phi_{j, k}(t), \psi_{j, k}(t)\right.$, onde $j$ e $\left.k \in Z\right\}$,

permite que a série ondaleta seja reescrita da seguinte forma:

$f(t)=\sum_{k} d_{j_{0}, k} \cdot \phi_{j_{0}, k}(t)+\sum_{j \geq j_{0}} \sum_{k} c_{j, k} \cdot \psi_{j, k}(t)$,

onde seus respectivos coeficientes $\left(d_{j_{0}, k}\right.$ e $\left.c_{j, k}\right)$ são dados por:

$c_{j, k}=\int_{-\infty}^{\infty} f(t) \cdot \psi_{j, k}(t) d t$

e

$d_{j_{0}, k}=\int_{-\infty}^{\infty} f(t) \cdot \phi_{j_{0}, k}(t) d t$

Um dos casos mais antigos, e também mais simples de ondaleta, é a ondaleta Haar. Sua função indicatriz, $\phi(t)$, é dada por:

$\phi(t)=\left\{\begin{array}{lr}0, & -\infty<t<0 \\ 1, & 0 \leq t<1 \\ 0, & 1 \leq t<\infty\end{array}\right.$

Cuja a base ondaleta pode ser construída a partir das Eqs.

(5) e (10), gerando a seguinte família ortogonal:

$\phi_{j, k}(t)=\left\{\begin{array}{l}2^{j / 2}, \text { se } \frac{k}{2^{j}} \leq t<\frac{k+1}{2^{j}} \\ 0, \text { para outros casos. }\end{array}\right.$

A função ondaleta de Haar $\psi(t)$ é definida por:

$\psi(t)=\left\{\begin{array}{rc}0, & -\infty<t<0 \\ 1, & 0 \leq t<1 / 2 \\ -1, & 1 / 2 \leq t<1 \\ 0, & 1 \leq t<\infty .\end{array}\right.$

Combinando-se as Eqs. (1) e (12), é possível construir a base ondaleta Haar:

$\psi_{j, k}(t)=\left\{\begin{array}{c}2^{j / 2}, \text { se } \frac{k}{2^{j}} \leq t<\frac{k+1 / 2}{2^{j}} \\ -2^{j / 2}, \text { se } \frac{k+1 / 2}{2^{j}} \leq t<\frac{k+1}{2^{j}} \\ 0, \text { para os outros casos. }\end{array}\right.$

O algoritmo piramidal (também conhecido como Transformada Discreta Wavelet, TDW), permite calcular os coeficientes da expansão de forma recursiva, reaproveitando os coeficientes $\left(d_{j, k}\right)$ da escala $j=0$ da base $\phi_{j, k}$ para obter as escalas posteriores através das seguintes equações:

$d_{j+1, k}=\frac{1}{\sqrt{2}}\left(d_{j, 2 k}+d_{j, 2 k+1}\right)$ $c_{j+1, k}=\frac{1}{\sqrt{2}}\left(d_{j, 2 k}-d_{j, 2 k+1}\right)$.

A Fig. 1 representa 0 algoritmo piramidal utilizado para obter os coeficientes de uma função $f(x)$, utilizando as Eqs. (14) e (15), partindo-se da escala $l=0 \mathrm{com}$ um número de amostras $N_{\max }+1$, onde $N_{\max }=7=2^{3}-1$.

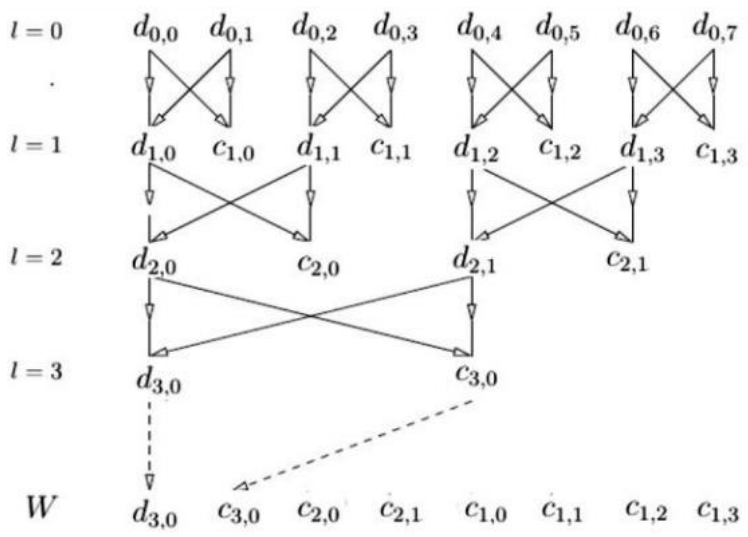

Figura 1: llustração gráfica do algoritmo piramidal.

\section{Metodologia}

Os campos de velocidades compressionais são funções bidimensionais, e a parametrização dos respectivos modelos são realizadas a partir da Eq. (7), que é utilizada para representar funções de uma única variável, enquanto os campos de velocidades são funções de duas variáveis. Para contornar esta situação é utilizada uma estratégia para coletar as velocidades $v(x, z)$ tornando-as funções de uma única variável $v(x)$. A malha utilizada na representação dos campos de velocidades é similar à mostrada na Fig. 2, onde considera-se um modelo geológico composto por linhas e colunas (similar a uma matriz), que é parametrizado linha por linha do modelo e depois os valores obtidos são colocados na posição de origem.

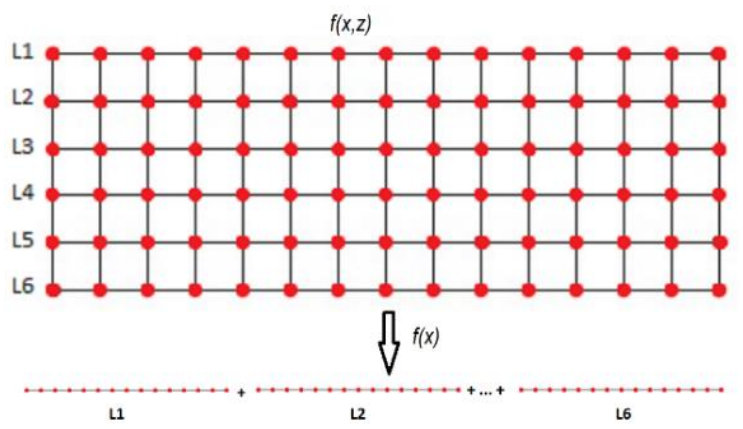

Figura 2: Malha regular utilizada na parametrização dos campos de velocidades sísmicas compressionais.

A parametrização de campos de velocidades compressionais, muitas vezes, utiliza uma grande quantidade de coeficientes, apesar do uso do algoritmo piramidal na obtenção dos mesmos. Sendo assim, duas alternativas são apresentadas, com a finalidade de excluir (ou igualar) parâmetros que exercem pouca, nenhuma ou aproximadamente a mesma influência na representação 
do modelo: a filtragem através de filtro passa-alta e a substituição por médias intervalares.

O filtro passa-alta elimina coeficientes inferiores a um ponte de corte $P c$ dado por:

$P c=10^{p}$

Portanto, todo coeficiente cujo módulo é menor que $10^{p}$ é desprezado.

Na substituição por médias intervalares, os coeficientes são organizados em ordem crescente e em cada intervalo $I_{i}$ se considera existir apenas um coeficiente que é dado pela média dos coeficientes de maior valor $\left(c_{\max , i}\right)$ e o de menor $\left(c_{\min , i}\right)$ no intervalo, a saber:

$\overline{c_{l}}=\frac{c_{\max , i}+c_{\min , i}}{2}$,

onde o índice $i$ representa o i-ésimo intervalo.

\section{Resultados}

As parametrizações foram realizadas com auxilio do algoritmo piramidal que gera coeficientes que permitem a representação de campos de velocidades sísmicas, que podem ser vistos como uma função discretizada do tipo $v(x, z)$. Utiliza-se a série ondaleta dada pela Eq. (7) e, posteriormente, técnicas de redução de coeficientes são aplicadas visando diminuir o número de parâmetros.

\section{Modelo de camadas horizontais}

A Fig. 3 mostra um modelo numérico de camadas horizontais, $M_{N 1}$. Tal modelo é dotado de várias propriedades físicas, tais como: densidade, porosidade, resistividade e etc. Entretanto, considerou-se apenas a propriedade da velocidade de propagação de ondas sísmicas compressionais, que é dada em termos numéricos e matriciais, e é utilizada como referência para a representação pela série ondaleta Haar.

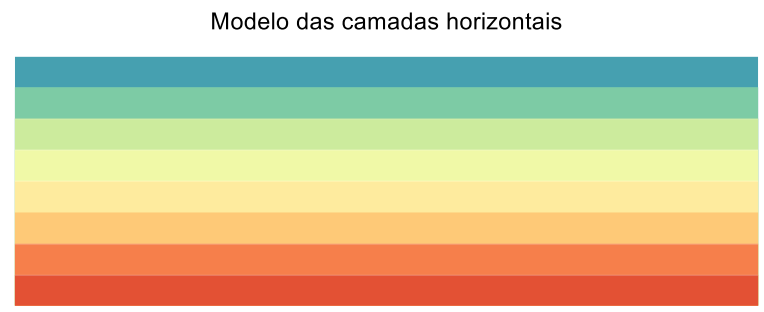

Figura 3: Modelo numérico do campo de velocidades sísmicas compressionais, $M_{N 1}$, utilizado na geração dos modelos parametrizados.

Aplicando-se o algoritmo piramidal para a obtenção de coeficientes, a partir do modelo numérico $M_{N 1}$, gera-se o modelo parametrizado $M_{P 1}$, mostrado na Fig. 4 .

Após utilizar os coeficientes fornecidos pelo algoritmo piramidal para parametrizar o modelo de camadas horizontais, as técnicas de redução de coeficientes, desenvolvidas em código fortran 95, foram aplicadas no modelo parametrizado. No primeiro resultado, representado na Fig. 5, utilizou-se o filtro corta-baixa, gerando assim o modelo parametrizado $\boldsymbol{M}_{\boldsymbol{P} 1 \boldsymbol{C}}$ e no segundo, obtido através da técnica de substituição por médias intervalares, é representado pelo modelo parametrizado $\boldsymbol{M}_{\boldsymbol{P 1} \boldsymbol{M}}$ e é mostrado na Fig. 6 .
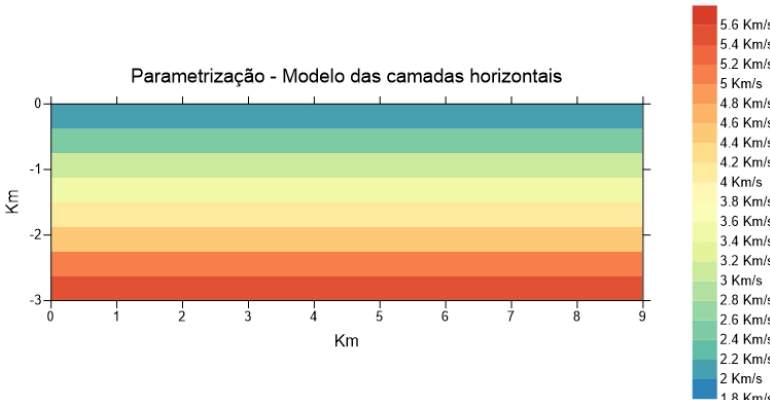

Figura 4: Modelo $M_{P 1}$, obtido através da parametrização de $M_{N 1}$ por ondaleta Haar, sem aplicação de técnicas de redução de coeficientes, utilizando-se índices $j$ compreendidos entre $j_{0}=1 \mathrm{e}$ $j_{\max }=16$, índices $k$ entre $k_{\min }=1$ e $k_{\max }=32768$ e com 8 coeficientes não-nulos.

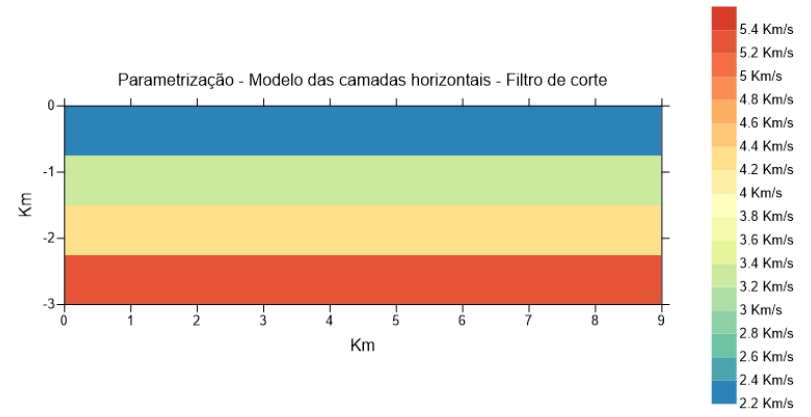

Figura 5: Modelo $M_{P 1 C}$ parametrizado por ondaleta Haar utilizando-se o filtro passa-alta com pontos de corte igual a $10^{1,55}$ e com 4 coeficientes não-nulos.

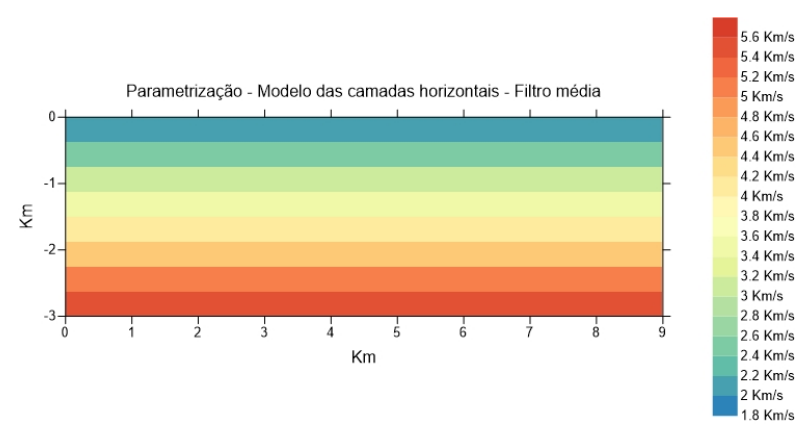

Figura 6: Modelo $M_{P 1 M}$ parametrizado por ondaleta $\mathrm{Haar}$ utilizando a técnica de substituição por médias intervalares e com 4 coeficientes não nulos.

\section{Modelo da intrusão ígnea}

A Fig. 7 mostra o modelo esquemático numérico da intrusão ígnea, $M_{N 2}$, um modelo que exibe um nível de complexidade um pouco mais elevado do que o anterior. Tal modelo é utilizado como referência para a aplicação das diferentes técnicas de redução de parâmetros aqui propostas.

Aplicando-se, novamente, o algoritmo piramidal, agora para o modelo numérico da intrusão ígnea $\left(M_{N 2}\right)$, obtêmse a parametrização deste modelo.

Após utilizar os coeficientes obtidos a partir do algoritmo piramidal para parametrizar o modelo $M_{N 2}$, técnicas de redução de coeficientes foram aplicadas no 
modelo parametrizado. O primeiro resultado, representado pela Fig. 9, utiliza o filtro corta-baixa, gerando assim o modelo parametrizado $M_{P 2 C}$ e o segundo, que utilizou a substituição por médias intervalares, é representado pelo modelo parametrizado $M_{P 2 M}$, mostrado na Fig. 10.

Modelo da intrusão ígnea

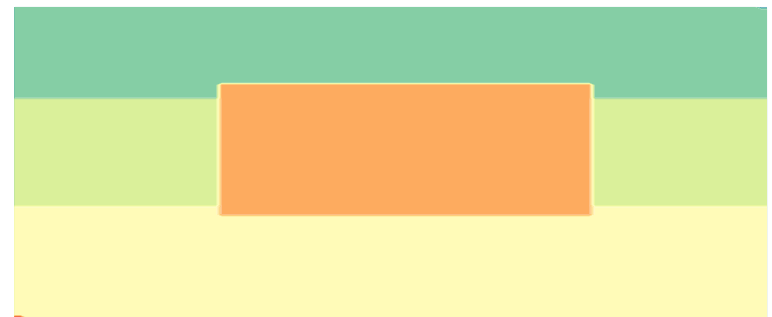

Figura 7: Modelo numérico do campo de velocidades sísmicas compressionais, $M_{N 2}$, utilizado na geração dos modelos parametrizados.
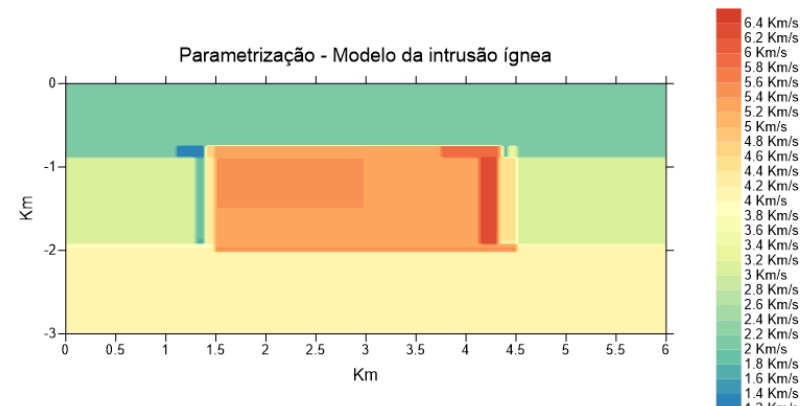

Figura 8: Modelo $M_{P 2}$, obtido através da parametrização de $M_{N 2}$ por série ondaleta Haar, sem uso de técnicas de redução de coeficientes, utilizando-se índices $j$ compreendidos entre $j_{0}=1 \mathrm{e}$ $j_{\max }=14$, índices $k$ entre $k_{\min }=1$ e $k_{\max }=8192$ e com 50 coeficientes não-nulos.
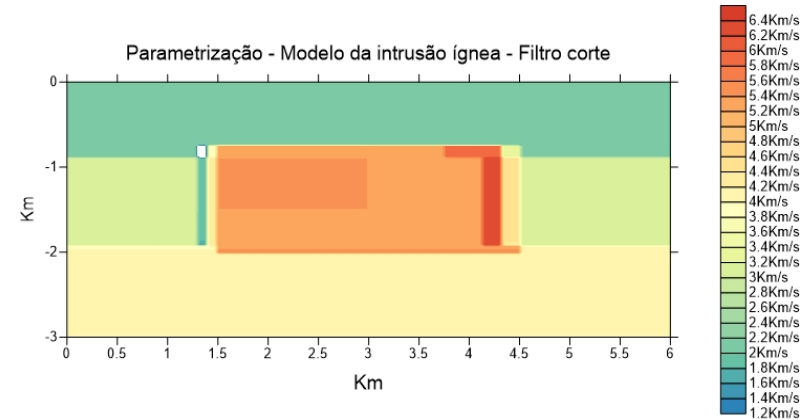

Figura 9: Modelo $M_{P 2 C}$ parametrizado por ondaleta Haar e utilizando o filtro passa-alta com pontos de corte igual a $10^{0,4}$ e com 21 coeficientes não-nulos.

\section{Conclusões}

A parametrização por série ondaleta Haar 1D, utilizando coeficientes obtidos através do algoritmo piramidal 1D, consegue representar satisfatoriamente um campo de velocidades sísmicas bidimensional utilizando um número pequeno de parâmetros.
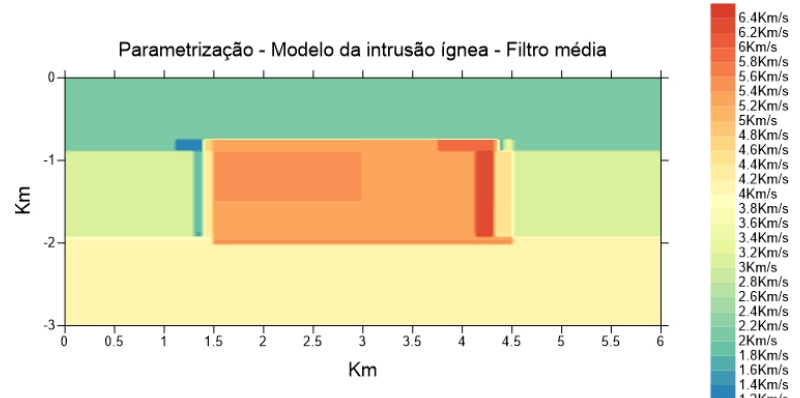

Figura 10: Modelo $M_{P 2 M}$ parametrizado por ondaleta Haar utilizando a técnica de redução de coeficientes por médias intervalares e com 20 coeficientes não nulos.

Apesar dos modelos utilizados serem simples, e utilizarem uma pequena quantidade de coeficientes na sua parametrização, esperava-se o uso de uma quantidade ainda menor de parâmetros. Sendo assim, técnicas de redução de coeficientes foram aplicadas: a filtragem passa alta e a substituição por médias intervalares.

As técnicas aplicada reduziram em cerca de 50\% (cinquenta por cento), ou mais, o número de coeficientes utilizados na parametrização por série ondaleta Haar.

A substituição por médias intervalares mostrou-se mais eficiente do que o filtro passa alta.

\section{Agradecimentos}

Os autores agradecem: ao CPGG-UFBA, ao CNPq e à Rede Temática de Estudos em Geofísica Aplicada da Petrobras pelo oferecimento de condições para a realização deste trabalho.

\section{Referências}

Cerqueira, A.G. \& Figueiró, W.M.; 2013. Campos de velocidades sísmicas do pré-sal e almofada de sal parametrizados por série de ondaleta Haar. Expanded Abstract, 13th International Congress of the Brazilian Geophysical Society, Rio de Janeiro, Brasil.

Cunha, P.E.M.; 2009. Uma introdução às bases teóricas para séries e transformadas de wavelets. Apostila.

Dos Santos, V.G.B. \& Figueiró, W.M.; 2011. Seismic ray tomography using $L 1$ integral norm. Revista Brasileira de Geofísica, RBGf, v. 29, n. 2, p. 347-358.

Martinez, A.C. \& Figueiró, W.M.; 2011. Parametrização ondaleta do campo de velocidades sísmicas relativo a um domo salino. Expanded Abstract, 12th International Congress of the Brazilian Geophysical Society (CISBGf), CDROM, Rio de Janeiro, RJ, Brasil.

Morettin, P.A.; 1999. Ondas e Ondaletas: da Análise de Fourier à Análise de Ondaletas. EDUSP.

Perin, H.M. \& Figueiró, W.M.; 2012. Parameterization of two-dimensional seismic velocity fields using the Haar wavelet. Society of Exploration Geophysicists (SEG), Technical Program, Expanded Abstracts, vol. 1, p. 1-5, SEG 82th Annual Meeting, Las Vegas, USA. 